\title{
Relaxation Dynamics and Interrupted Coarsening in Irrationally Frustrated Superconducting Arrays
}

\author{
Gun Sang Jeon, ${ }^{1}$ Sung Jong Lee, ${ }^{2}$ Bongsoo Kim, ${ }^{3}$ and M.Y. Choi ${ }^{1,4}$ \\ ${ }^{1}$ Department of Physics and Astronomy, Seoul National University, Seoul 151-747, Korea \\ ${ }^{2}$ Department of Physics, University of Suwon, Kyonggi-do 445-743, Korea \\ ${ }^{3}$ Department of Physics, Changwon National University, Changwon 641-773, Korea \\ ${ }^{4}$ Asia-Pacific Center for Theoretical Physics, Pohang 790-784, Korea
}

\begin{abstract}
Equilibrium and non-equilibrium relaxation behaviors of two-dimensional superconducting arrays are investigated via numerical simulations at low temperatures in the presence of incommensurate transverse magnetic fields, with frustration parameter $f=(3-\sqrt{5}) / 2$. We find that the nonequilibrium relaxation, beginning with random initial states quenched to low temperatures, exhibits a three-stage relaxation of chirality autocorrelations. At the early stage, the relaxation is found to be described by the von Schweidler form. Then it exhibits power-law behavior in the intermediate time scale and faster decay in the long-time limit, which together can be fitted to the Ogielski form; for longer waiting times, this crosses over to a stretched exponential form. We argue that the powerlaw behavior in the intermediate time scale may be understood as a consequence of the coarsening behavior, leading to the local vortex order corresponding to $f=2 / 5$ ground-state configurations. High mobility of the vortices in the domain boundaries, generating slow wandering motion of the domain walls, may provide mechanism of dynamic heterogeneity and account for the long-time stretched exponential relaxation behavior. It is expected that such meandering fluctuations of the low-temperature structure give rise to finite resistivity at those low temperatures; this appears consistent with the zero-temperature resistive transition in the limit of irrational frustration.
\end{abstract}

PACS numbers: $74.50+\mathrm{r}, 67.40 . \mathrm{Fd}$

\section{INTRODUCTION}

Relaxation properties of systems with a great number of metastable states have attracted much attention in the recent decades,,$\underline{1,2,3,4,5,6}$ These systems are usually characterized by the existence of both disorder and frustration. A well-known system is the Ising spin glass model,,$\underline{1}$ which displays non-exponential relaxation at low temperatures $\frac{2.3}{}$ Interesting aging phenomena ${ }^{4}$ were also observed in this system. $\frac{5}{5}$ The Coulomb glass model, which is another interesting disordered system, has recently been shown to exhibit relaxation of the stretched exponential form $\underline{\underline{6}}$ Such non-exponential relaxation was also reported in frustrated systems without disorder and its relation with the percolation transition was discussed ${ }^{7}$

In a superconducting array, frustration can be induced in a controllable way by applying an external magnetic field. It has crucial effects on thermodynamics of the system and results in a variety of equilibrium properties $\underline{8,9}$ Such remarkable diversity in the equilibrium properties naturally leads to expectations that a variety of interesting dynamic behaviors is also present, particularly in relaxation toward equilibrium.

An interesting limiting situation arises in the presence of irrational frustration, the most typical case of which is provided by the frustration parameter $f=1-g$ with the golden number $g \equiv(\sqrt{5}-1) / 2$. The system was first suggested to exhibit a spin-glass-like phase at low temperatures due to self-generated disorder $\stackrel{10}{=}$ Subsequently, it was argued to display novel finite-size effects 11 that the size-dependent transition temperature decreases mono- tonically with the system size, resulting in the absence of a finite-temperature transition $\stackrel{12}{=}$ Simulation results for the current-voltage characteristics supported the zerotemperature transition, 13 while experimental results were interpreted to exhibit finite-temperature transitions ${ }^{14.15}$ Recent Monte Carlo simulations, investigating the vortex configuration at low temperatures in the system with $f$ given by rational approximants to $1-g$, indicate the existence of a low-temperature phase where the helicity modulus takes a finite value along one direction and vanishes along the other down to very low temperatures $16,17,18$ From dynamical perspectives, due to the existence of many metastable states that are almost degenerate with one another (which is attributable to the incommensurate magnetic field), one may expect characteristic slow relaxation in the array with irrational frustration. Dynamic simulations of this system, based on simple Langevin dynamics, indeed disclosed a crossover temperature below which strongly nonexponential relaxation emerges, exhibiting some analogy to the behavior of supercooled liquids $\underline{19}$

In this paper we investigate the relaxation behavior of the superconducting array with irrational frustration in both equilibrium and nonequilibrium situations, employing the resistively-shunted-junction (RSJ) dynamics in the overdamped limit (i.e., junction capcitances are neglected). Note that the present RSJ dynamics can, in principle, be realized in real junction array experiments; this is in contrast to the Langevin dynamics employed in existing simulations, which assumes hypothetic dissipation between the superconducting islands and the ground. 
At equilibrium, we observe that chirality autocorrelations are characterized by stretched exponential relaxation (with a temperature-dependent stretching exponent) in a wide range of intermediate and low temperatures. The relaxation time exhibits non-Arrhenius behavior with the Vogel-Tammann-Fulcher type of temperature dependence.

On the other hand, beginning with random initial states quenched to low temperatures $(T<0.14)$, the dynamics exhibits slow aging behavior, not reaching the equilibrium relaxation within our computing time. It is observed that, for a short time (approximately up to time $t \approx 10$ to 20 depending on the waiting time and temperature), relaxation of the chirality autocorrelation function exhibits the von Schweidler behavior 20 After the short time, chirality autocorrelations for short waiting times relax according to the so-called Ogielski form with temperature-dependent exponents. For longer waiting times, this behavior slowly changes into the stretched exponential form with a temperature-dependent stretching exponent. The Ogielski form of the nonequilibrium relaxation is characterized by power-law behavior in the intermediate time scale and faster decay in the long-time region. The emergence of power-law behavior in the intermediate time scale suggests the presence of a sort of coarsening dynamics with dynamic scaling, which is supported by the evolution of vortex patterns.

This is also consistent with the vortex configurations in low-energy states obtained from a global optimization algorithm such as conformational space annealing (CSA) $\stackrel{21,22}{=}$ Those vortex configurations obtained from CSA exhibit interesting features: There exist approximately parallel domains of local vortex order corresponding to the staircase ground state of $f=2 / 5 \underline{\underline{23}}$ Those domains are separated by domain-wall regions, consisting of characteristic local arrangements of four consecutive vacancies along one (horizontal or vertical) direction, thereby neighboring domains are parallel-shifted by two horizontal (or vertical) lattice units. Reminiscent of the smectic order in a liquid crystal, such configurations may be described as smectic (liquid-crystalline) arrangements of diagonal chains of vortices. Similar features of the vortex lattice were also reported for the case of frustration $f=13 / 34$ and $21 / 55$, which are rational approximants to the irrational value $f=1-g \underline{\underline{18}}$

Evolution of the vortex pattern in general exhibits growth of local vortex order with time toward the lowtemperature anisotropic state described above. This power-law behavior of coarsening is expected to be interrupted by long-time fluctuations of domain walls, limiting the maximum local domain size to around 12 lattice units on average. In the long-time limit, vortex motions occur predominantly in the domain-wall region through transfer of vortices between neighboring diagonal vortex chains, which correspond also to the motion of four consecutive vacancies. Accordingly, the higher mobility of the vortices located in domain-wall regions can naturally explain dynamic heterogeneity in the system, leading also to the stretched exponential relaxation.

As for the resistive transition, one can argue that, as long as the domain-wall defects keep fluctuating with unbounded displacement, the system would remain resistive (i.e., exhibiting finite resistance) even at low temperatures below the transition to the anisotropic phase, since there always exists a direction along which the helicity modulus vanishes. Those domain-wall defects are likely to freeze at a much lower temperature which vanishes in the limit of irrational frustration; thus concluded is a zero-temperature resistive transition in the system with irrational frustration. Note, however, that this argument applies to a pure system with no quenched disorder. In a real Josephson-junction array, disorder is inevitable in the distribution of critical currents of individual junctions, which leads to pinning of domain-wall defects at finite temperatures. This may explain the recent experimental results reporting a finite-temperature resistive transition near incommensurability of the magnetic frustration $\frac{15}{15}$

This paper is organized as follows: In Sec. II we introduce the equations of motion for the RSJ dynamics of the system in the fluctuating twist boundary conditions. Section III presents the results of simulations performed on the equations of motion. Both equilibrium and nonequilibrium relaxation behaviors of chirality autocorrelations are examined and coarsening, interrupted by fluctuating domain-wall defects, is addressed. Finally, a summary is given in Sec. IV.

\section{EQUATIONS OF MOTION}

We begin with the set of equations of motion for the phases $\left\{\phi_{i}\right\}$ of the superconducting order parameters in an $L \times L$ square array. In the RSJ model under the

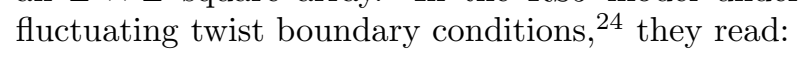

$$
\sum_{j}^{\prime}\left[\frac{d \widetilde{\phi}_{i j}}{d t}+\sin \left(\widetilde{\phi}_{i j}-\mathbf{r}_{i j} \cdot \boldsymbol{\Delta}\right)+\zeta_{i j}\right]=0
$$

where we have employed the abbreviations $\widetilde{\phi}_{i j} \equiv$ $\phi_{i}-\phi_{j}-A_{i j}$ and $\mathbf{r}_{i j} \equiv \mathbf{r}_{i}-\mathbf{r}_{j}$, and the primed summation runs over the nearest neighbors of grain $i$. The position of grain $i$ is represented by $\mathbf{r}_{i}=\left(x_{i}, y_{i}\right)$ with the lattice constant set equal to unity while the gauge field $A_{i j}$ is given by the line integral of the vector potential $\mathbf{A}$ :

$$
A_{i j} \equiv \frac{2 \pi}{\Phi_{0}} \int_{\mathbf{r}_{i}}^{\mathbf{r}_{j}} \mathbf{A} \cdot d \mathbf{l}
$$

with the flux quantum $\Phi_{0} \equiv h c / 2 e$. The frustration parameter $f$, which measures the number of flux quanta per plaquette, is given by the directional sum of the gauge field $A_{i j}$ around a plaquette:

$$
f \equiv \frac{1}{2 \pi} \sum_{P} A_{i j}
$$


In Eq. (11) the energy and the time have been expressed in units of $\hbar I_{c} / 2 e$ and $\hbar / 2 e R I_{c}$, respectively, with singlejunction critical current $I_{c}$ and shunt resistance $R$. The thermal noise current $\zeta_{i j}$ is assumed to be white, satisfying

$$
\left\langle\zeta_{i j}(t+\tau) \zeta_{k l}(t)\right\rangle=2 k_{B} T \delta(\tau)\left(\delta_{i k} \delta_{j l}-\delta_{i l} \delta_{j k}\right)
$$

at temperature $T$. Henceforth we set the Boltzmann constant $k_{B} \equiv 1$, thus measuring the temperature in units of $\hbar I_{c} / 2 e k_{B}$. The dynamics of the twist variables $\boldsymbol{\Delta} \equiv\left(\Delta_{x}, \Delta_{y}\right)$ is governed by

$$
\frac{d \Delta_{a}}{d t}=\frac{1}{L^{2}} \sum_{\langle i j\rangle_{a}} \sin \left(\widetilde{\phi}_{i j}-\Delta_{a}\right)+\zeta_{a}
$$

where $\sum_{\langle i j\rangle_{a}}$ denotes the summation over all nearestneighboring pairs in the $a$-direction $(a=x, y)$ and $\zeta_{a}$ satisfies

$$
\left\langle\zeta_{a}(t+\tau) \zeta_{b}(t)\right\rangle=\frac{2 T}{L^{2}} \delta_{a b} \delta(\tau) .
$$

To study the relaxation of the system, we let the system evolve from some random initial configurations quenched to given temperatures and measure the chirality autocorrelation function:

$$
C_{q}\left(t+t_{w}, t_{w}\right) \equiv \frac{1}{L^{2} f(1-f)} \sum_{\mathbf{R}}\left\langle q_{\mathbf{R}}\left(t+t_{w}\right) q_{\mathbf{R}}\left(t_{w}\right)\right\rangle
$$

with the waiting time $t_{w}$. Here the chirality is defined to be

$$
q_{R}(t) \equiv \frac{1}{2 \pi} \sum_{P}\left[\widetilde{\phi}_{i j}(t)-\mathbf{r}_{i j} \cdot \boldsymbol{\Delta}(t)\right],
$$

where $\sum_{P}$ denotes the directional plaquette summation of links around dual lattice site $\mathbf{R}$ and the phase difference $\widetilde{\phi}_{i j}(t)-\mathbf{r}_{i j} \cdot \boldsymbol{\Delta}(t)$ is defined modulo $2 \pi$ in the range $(-\pi, \pi]$.

In numerical simulations, we have integrated directly the equations of motion (11) and (5) via the modified Euler method with time step $\Delta t=0.05$. The time step has been varied, only to give no appreciable difference. We have considered mostly systems of linear size $L=55$ and 89, taking averages typically over 100 to 600 ensembles with random initial states. The size has been chosen as members of the Fibonacci sequence, thus to minimize the boundary effects due to the irrational frustration $f=$ $1-g$. For comparison, we have also considered close rational approximants $f=21 / 55$ and $34 / 89$, to find no qualitative difference in relaxation dynamics.

\section{SIMULATION RESULTS}

We first consider the equilibrium relaxation behavior of the system, obtained in the following way: Dynamic simulations are performed with random initial states and then the autocorrelation function in Eq. (7) is computed for different values of the waiting time. When the waiting time is sufficiently large (and the temperature is not too low), the autocorrelation function no longer depends on the waiting time, collapsing onto a single relaxation function. This collapsed relaxation function is taken to be the equilibrium relaxation function $C_{e q}(t)$. In this way, equilibration of the system is achieved here, down to temperature $T=0.14$. In Fig. 1(a), the behavior of $C_{e q}(t)$ is exhibited for several values of the temperature $T$. Excluding the earliest time regime, one may fit this relaxation behavior to a stretched exponential form: $C_{e q}(t) \approx A \exp \left[-(t / \tau)^{\beta}\right]$. Figure 1(b) shows that the stretching exponent $\beta$ decreases as the temperature is lowered, reaching the value $\beta \approx 0.5$ at $T=0.14$. On the other hand, Fig. 1(c), plotting the relaxation time $\tau$ versus the inverse temperature $T^{-1}$, discloses the VogelTamman-Fulcher behavior:

$$
\tau(T)=\tau_{0} \exp \left[D \frac{T_{0}}{T-T_{0}}\right]
$$

with the fragility parameter $D=10.2$ and other parameters $\tau_{0}=3.65$ and $T_{0}=0.06$. (Here $T_{0}$ is merely a fitting parameter, perhaps not associated with a transition.) These results are consistent with those from Langevin dynamics $\frac{19}{19}$

As the temperature is further lowered, especially below 0.14 , the system, starting from a random initial state, does not relax to the equilibrium within the available computing time. Instead of pursuing equilibrium relaxation at these low temperatures, we probe nonequilibrium relaxation for various waiting times by letting the system evolve from random initial states. It is found that the resulting relaxation of chirality autocorrelations proceeds in three stages. Figure 2 (a) shows the time evolution of $C_{q}\left(t+t_{w}, t_{w}\right)$ for the waiting time $t_{w}=30,000$ at temperature $T=0.15,0.13$, and 0.10 . For the same data, plotted in Fig. 2(b) is $1-C_{q}\left(t+t_{w}, t_{w}\right)$ versus time $t$, where one can see that, for almost three decades beginning from the earliest time, the relaxation fits nicely to the von Schweidler form $1-\left(t / \tau_{0}\right)^{b}$ with $b \approx 1 / 2$. In Fig. 2(c) we show $1-C_{q}\left(t+t_{w}, t_{w}\right)$ versus time $t$ at temperature $T=0.13$ for various waiting times. It is observed that the von Schweidler behavior with $b \approx 1 / 2$ is rather robust, hardly depending on the waiting time or the target temperature. This is in contrast with the results of Langevin simulations, where the value of $b$ tends to deviate from $1 / 2$, getting smaller at low temperatures. 19 We presume that this discrepancy arises from the difference in vortex dynamics (and diffusion) at short times between RSJ dynamics and simple Langevin dynamics.

We now turn to the relaxation behavior at intermediate and late stages for various waiting times and temperatures. Figure 3 shows the nonequilibrium relaxation of the chirality autocorrelation function for null waiting time at temperature $T=0.15,0.14,0.13$, and 0.12 . It is 
observed that the relaxation exhibits power-law behavior in the intermediate-time regime, followed by faster decay in the long-time regime. Here it is tempting to fit the relaxation of the autocorrelation function to the Ogielski form:

$$
C_{q}(t) \approx A_{1} t^{-\alpha} \exp \left[-\left(t / \tau_{1}\right)^{\beta}\right]
$$

with the exponents $\alpha$ and $\beta$ given in Fig. 4 and Table I. Note that $\alpha$ depends substantially on the temperature, varying in the range of 0.19 to 0.35 at temperatures between 0.08 and 0.15 .

\begin{tabular}{ccc}
\hline \hline$T$ & $\alpha$ & $\beta$ \\
\hline 0.08 & $0.19(1)$ & - \\
0.09 & $0.23(1)$ & - \\
0.10 & $0.258(15)$ & $1.27(15)$ \\
0.11 & $0.264(10)$ & $0.80(3)$ \\
0.12 & $0.300(15)$ & $0.67(3)$ \\
0.13 & $0.300(13)$ & $0.54(2)$ \\
0.14 & $0.350(10)$ & $0.83(4)$ \\
0.15 & $0.350(10)$ & $0.83(4)$ \\
\hline \hline
\end{tabular}

TABLE I: Exponents $\alpha$ and $\beta$ depending on the temperature $T$. Note that $\beta$ is not shown for the cases of $T=0.08$ and 0.09 . At these low temperatures, relaxation is too slow to observe clearly the late-time stretched exponential part within the computational time window, making it formidable to estimate $\beta$.

Also shown in Fig 5 is the nonequilibrium relaxation of the chirality autocorrelation function for various waiting times, at temperature $T=0.14,0.13$, and 0.12 . For longer waiting times $\left(t_{w}>100.0\right)$, this behavior crosses over to the stretched exponential form $C_{q}(t) \approx$ $A_{2} \exp \left[-\left(t / \tau_{2}\right)^{\beta}\right]$. Emergence of the power-law behavior of the nonequilibrium relaxation at intermediate times strongly suggests that there exist some coarsening processes in the system. Figure 6 exhibits snapshots of the vortex configuration at temperature $T=0.13$, taken at several time instants; one can recognize slow growth of local order corresponding to $f=2 / 5$ vortex patterns, with diagonal chain structures. Since the frustration of the system is given by $f=1-g$, which is slightly smaller than $2 / 5$, these locally ordered domains of $f=2 / 5$ patterns may not grow to span the whole system. Instead, there should exist finite length (and also time) scales for the growth of these local domains, beyond which the growth is interrupted by domain-wall regions of lower vortex density, so that the net vortex density of the whole system becomes equal to $1-g$.

In order to understand the vortex configuration attained in the long-time limit at low temperatures, we investigate the configuration of low-energy states by means of the efficient optimization algorithm, CSA ${ }^{21}$ Snapshots of typical vortex configurations obtained via CSA are shown in Fig 7, where we observe domains of locally ordered vortex patterns corresponding to the staircase ground state of the system with frustration (or vortex density) $f=2 / 5$. These domains of typical width 9 to 12 lattice spacings are separated by domain-wall (line defect) regions that consist of characteristic local arrangements of four neighboring vacancies (see bar-shaped regions, each with four consecutive empty plaquettes); there the vortex density is lower than the locally ordered regions (of local vortex density $2 / 5$ ) in such a way that the net vortex density of the whole system is precisely equal to $1-g$ (which is less than 2/5). This configurations may also be described as a liquid-crystal-type arrangement of diagonal chains of vortices (of length 9 to 12), with the neighboring chains of vortices shifted in the diagonal direction by about half the length of the chains. Similar configurations were observed in the case of rational approximants to $1-g \underline{\underline{18}}$

In the long-time limit, we expect that the coarsening dynamics will lead eventually to the locally ordered configuration with domain walls, shown in Fig. 7. Within a locally ordered domain, vortices are almost rigid and resist moving. In contrast, those vortices at ends of the chains are easily put in motion, jumping into the barshaped vacancies in domain-wall regions and thus joining another vortex chain. This in turn gives rise to domain-wall fluctuations (or equivalently, fluctuations in the length of the vortex chains), which would lead slowly but ultimately to the complete restructuring of the local vortex configuration. It is thus expected that dynamic heterogeneity naturally emerges from the existence of distinctly mobile vortices in the domain-wall regions ${ }^{25}$

We believe that such domain-wall fluctuations and restructuring of the local vortex configuration can explain the absence of freezing of the relaxation at low temperatures. Namely, the low-temperature state is presumably of a liquid crystalline type: Even though there exists orientational order in the chain-like arrangement of vortices, those diagonal chains can flow (like a liquid) due to the transfer of vortices between neighboring chains, corresponding to the fluctuating motion of the bar-shaped vacancies and resulting in finite resistivity. At a much lower temperature one may expect complete freezing of the domain walls to occur. The freezing temperature should depend on the commensurability of the distribution of the vacancy defects and the underlying background vortex lattice; this is directly related to the rationality of $f=p / q$ (with $p$ and $q$ relatively prime integers) and the freezing temperature is expected to vanish in the irrational limit $(q \rightarrow \infty)$.

It is of some interest to note the similarity to the behavior found in the lattice coulomb gas with charge density near the golden number ${ }^{26}$ Even though the detailed ordering pattern is different, it was found numerically that there exist two-step transitions in the lattice coulomb gas with $1 / 3<f<2 / 5$, where the intermediate phase corresponds to anisotropic striped charge ordering together with mobile charges within partially-filled channels. There the lower transition corresponds to the complete freezing of charges within partially filled diag- 
onal channels, which occurs at temperatures sensitively dependent on the rationality of $f$ due to the commensurability effects. In this study our system is the Josephsonjunction array in the limit of irrational frustration, and we thus expect that the true vortex freezing would occur at zero temperature.

As long as the domain-wall defects fluctuate with unbounded displacement, the system should remain resistive, exhibiting finite resistance. These domain-wall defects are expected to freeze at much lower temperature of the order $1 / q$ for $f=p / q$; this leads to the zerotemperature resistive transition in the limit of irrational frustration. Note that this argument applies for pure systems with no quenched disorder. In the case of real Josephson-junction arrays, some type of disorder, e.g., in the distribution of the critical currents of individual junctions is unavoidable. Therefore pinning of the domainwall defects can easily occur at finite temperatures, having resistance vanish. This may explain the recent experiment reporting a finite-temperature resistive transition near incommensurability of the magnetic frustration, where disorder in the critical currents of individual junctions was noted to be up to $15 \%, \frac{15}{=}$

\section{SUMMARY}

We have studied the relaxation behavior of the chirality autocorrelation function in two-dimensional superconducting arrays under irrational frustration at intermediate and low temperatures. Both equilibrium relaxation and nonequilibrium relaxation have been investigated via numerical simulations of RSJ dynamics. Equilibrium relaxation dynamics reveals characteristic fea- tures of the stretched exponential form with the VogelTamman-Fulcher dependence of the relaxation time.

Nonequilibrium relaxation at low temperatures, beginning with random initial states exhibits interesting waiting-time dependence: For short waiting times, the relaxation follows a power-law behavior in the intermediate-time regime and faster decay in the longtime regime, characterized together by the Ogielski form with temperature dependent exponents. For longer waiting times, this gradually crosses over to a stretched exponential form. Further, in all cases of short and long waiting times, the relaxation at early time stage fits nicely to the von Schweidler form with exponent about $1 / 2$.

It has been argued that the power-law behavior originates from coarsening dynamics up to a certain length scale, with the local vortex order corresponding to the ground state of $f=2 / 5$, found to be consistent with the vortex configurations of low-energy states. This coarsening, however, is interrupted by the presence of domainwall defects. Such chain-like domain-wall regions are expected to provide possible mechanism of dynamic heterogeneity and stretched exponential relaxation. Since the RSJ dynamics adopted in this study can be realized experimentally, it would be of interest to carry out experiment and compare the results.

\section{Acknowledgments}

This work was supported in part by the BK21 Project. M.Y.C. also acknowledges a visitor grant from the CNRS, France and thanks the Laboratoire de Physique Théorique, Strasbourg, for its kind hospitality during his stay.
${ }^{1}$ K. Binder and A.P. Young, Rev. Mod. Phys. 58, 801 (1986).

2 W.L. McMillan, Phys. Rev. B 28, 5216 (1983).

3 A.T. Ogielski, Phys. Rev. B 32, 7384 (1985).

4 L. Lundgren, P. Svedlindh, P. Nordblad, and O. Beckman, Phys. Rev. Lett. 51, 911 (1983).

${ }^{5}$ H. Rieger, Physica A 224, 267 (1996); J. Phys. A 26, L615 (1993).

6 A. P'erez-Garrido, M. Ortuño, A. Díaz-Sánchez, and E. Cuevas, Phys. Rev. B 59, 5328 (1999); A. Díaz-Sánchez and A. Pérez-Garrido, Eur. Phys. J. B 24, 483 (2001).

7 A. Fierro, G. Franzese, A. de Candia, and A. Coniglio, Phys. Rev. E 59, 60 (1999); G. Franzese, arxiv.org: cond-mat/0107491.

8 S. Teitel and C. Jayaprakash, Phys. Rev. Lett. 51, 1999 (1983).

9 See, e.g., Macroscopic Quantum Phenomena and Coherence in Superconducting Networks, edited by C. Giovannela and M. Tinkham (World Scientific, Singapore, 1996); ICTP Workshop on Josephson Junction Arrays, edited by H.A. Cerdeira and S.R. Shenoy [Physica B 222, 253 (1996)].
10 T.C. Halsey, Phys. Rev. Lett. 55, 1018 (1985).

11 M.Y. Choi, J.S. Chung, D. Stroud, and J. Choi, Phys. Rev. B 40, 5147 (1989); S.Y. Park, M.Y. Choi, B.J. Kim, G.S. Jeon, and J.S. Chung, Phys. Rev. Lett. 85, 3484 (2000); G.S. Jeon and M.Y. Choi, Physica A 315, 255 (2002).

12 M.Y. Choi and D. Stroud, Phys. Rev. B 32, 7532 (1985); ibid. 35, 7109 (1987).

13 E. Granato, Phys. Rev. B 54, R9655 (1996); Braz. J. Phys. 32, 699 (2002); arXiv:0704.1097.

14 F. Yu, N.E. Israeloff, A.M. Goldman, and R. Bojko, Phys. Rev. Lett. 68, 2535 (1992).

15 Y.-J. Yun, I.-C. Baek, and M.-Y. Choi, J. Korean Phys. Soc. 37, 626 (2000); I.-C. Baek, Y.-J. Yun, and M.-Y. Choi, Physica C 388, 785 (2003); Phys. Rev. B 69, 172501 (2004).

16 P. Gupta, S. Teitel, and M.J.P. Gingras, Phys. Rev. Lett. 80, 105 (1998).

17 C. Denniston and C. Tang, Phys. Rev. B. 60, 3163 (1999); M.R. Kolahchi, ibid. 59, 9569 (1999).

18 M.R. Kolahchi and H. Fazli, Phys. Rev. B 62, 9089 (2000).

19 B. Kim and S.J. Lee, Phys. Rev. Lett. 78, 3709 (1997).

20 W. Götze, J. Phys.: Condens. Matter 11, A1 (1999). 
21 J. Lee, H.A. Scheraga, and S. Rackovsky, J. Comput. Chem. 18, 1222 (1997); Biopolymers 46, 103 (1998); J. Lee and H.A. Scheraga, Int. J. Quantum Chem. 75, 255 (1999).

22 J. Lee, I.-H. Lee, and J. Lee, Phys. Rev. Lett. 91, 080201 (2003).

23 T.C. Halsey, Phys. Rev. B 31, 5728 (1985).
24 B.J. Kim, P. Minnhagen, and P. Olsson, Phys. Rev. B 59, 11506 (1999).

25 D. N. Perera and P. Harrowell, Phys. Rev. Lett. 81, 120 (1998).

26 S.J. Lee, J.-R. Lee, and B. Kim, Phys. Rev. Lett. 88, 025701 (2002). 


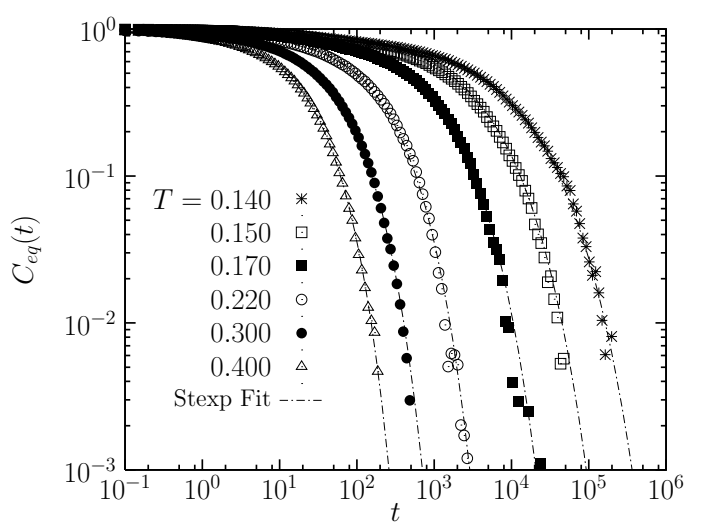

(a)

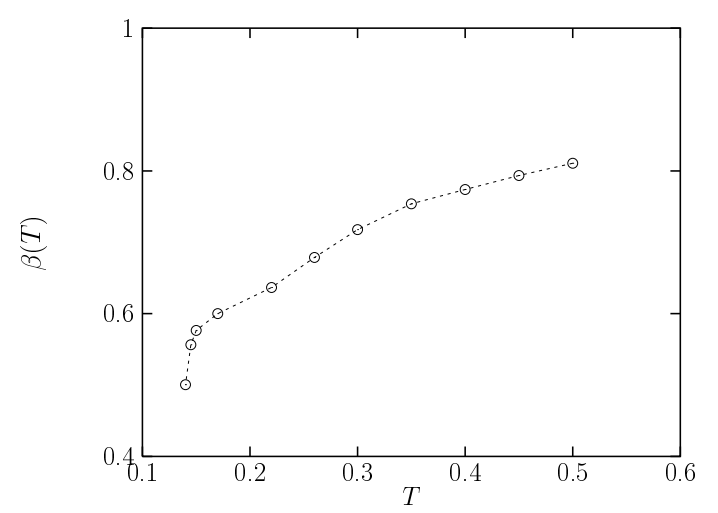

(b)

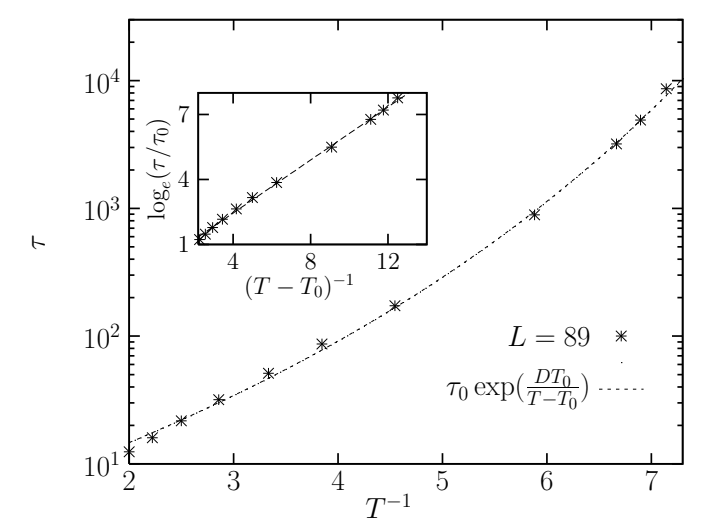

(c)

FIG. 1: (a) Relaxation of the chirality autocorrelation function (at equilibrium) $C_{e q}(t)$ at various temperatures $T$ in the irrationally frustrated array of linear size $L=89$. Stretched exponential fits are also plotted. (b) Stretching exponent versus the temperature. The dotted line is merely a guide to the eye. (c) Relaxation time versus the inverse temperature. The dashed line represents the Vogel-Tammann-Fulcher fit with $T_{0}=0.06$ and $\tau_{0}=3.65$. The inset shows the result in a different scale. 


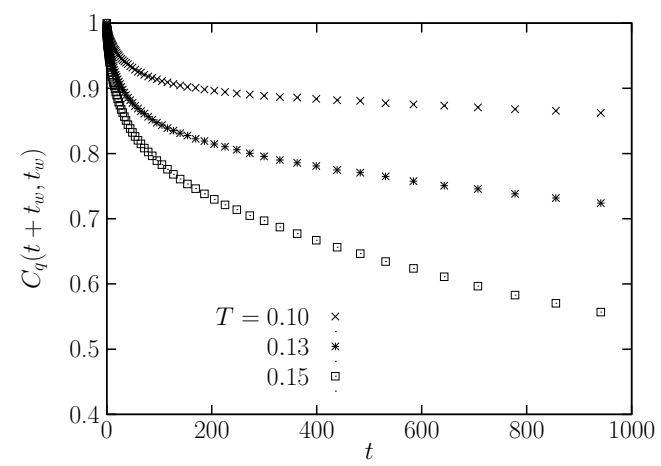

(a)

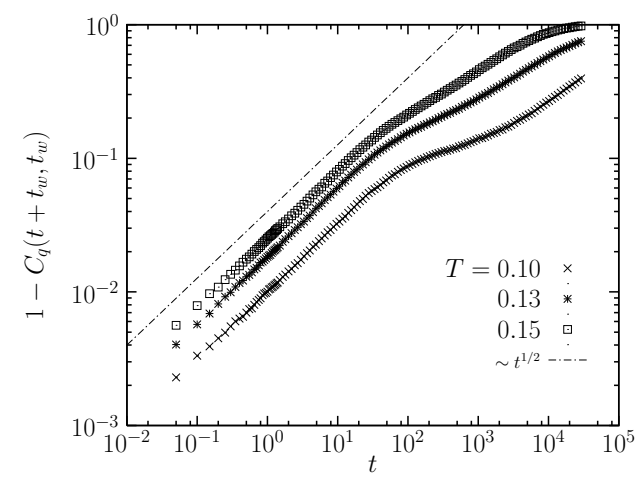

(b)

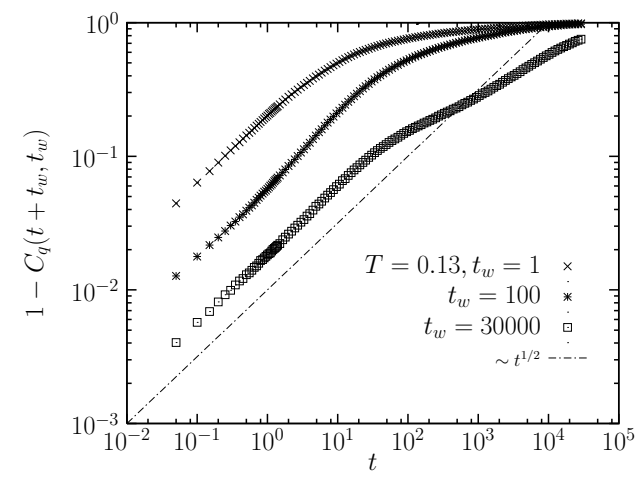

(c)

FIG. 2: (a) Relaxation of the chirality autocorrelation function $C_{q}\left(t+t_{w}, t_{w}\right)$ with time $t$ in the early-time regime at temperatures $T=0.10,0.13$, and 0.15 for $L=89$. The waiting time is chosen to be $t_{w}=30000$. (b) The log-log plot of $1-C_{q}\left(t+t_{w}, t_{w}\right)$ versus $t$ for the same data. For almost three decades in the early-time regime, the relaxation behavior is observed to follow $1-C_{q}\left(t+t_{w}, t_{w}\right) \sim t^{1 / 2}$ at all the three temperatures, as indicated by the dot-dashed line. (c) The log-log plot of $1-C_{q}\left(t+t_{w}, t_{w}\right)$ versus $t$ at temperature $T=0.13$ for three different waiting times $t_{w}=1.0,100$ and 30000 . It is shown that the early-time behavior does not depend qualitatively on the waiting time. 


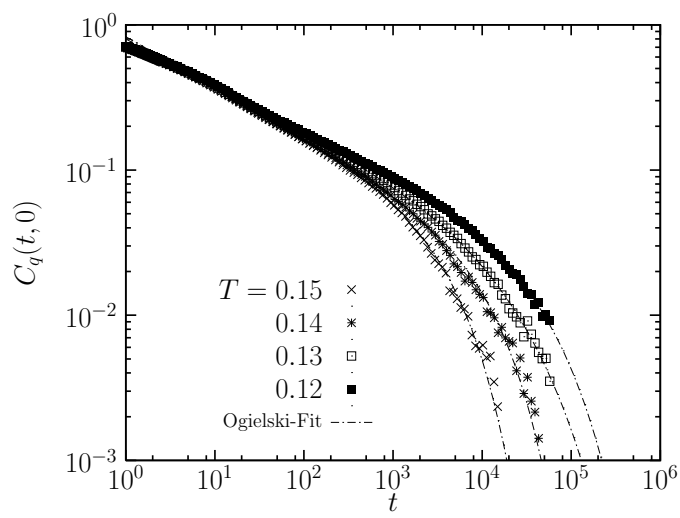

FIG. 3: Nonequilibrium relaxation of the chirality autocorrelation function $C_{q}\left(t+t_{w}, t_{w}\right)$ for zero waiting time $\left(t_{w}=\right.$ $0.0)$, starting from random initial states, at temperature $T=0.15,0.14,0.13$ and 0.12 . While in the intermediate-time regime the nonequilibrium relaxation function exhibits features of power-law behavior, this behavior is interrupted by faster decay in the long-time regime, which together can be fitted to the Ogielski form, as shown by the dot-dashed lines.

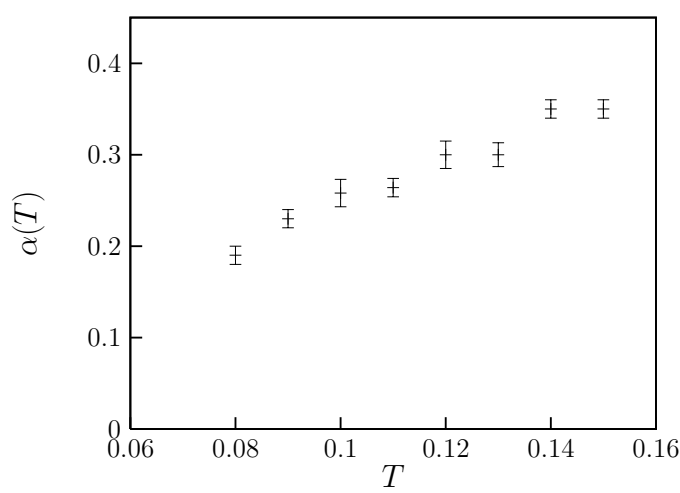

(a)

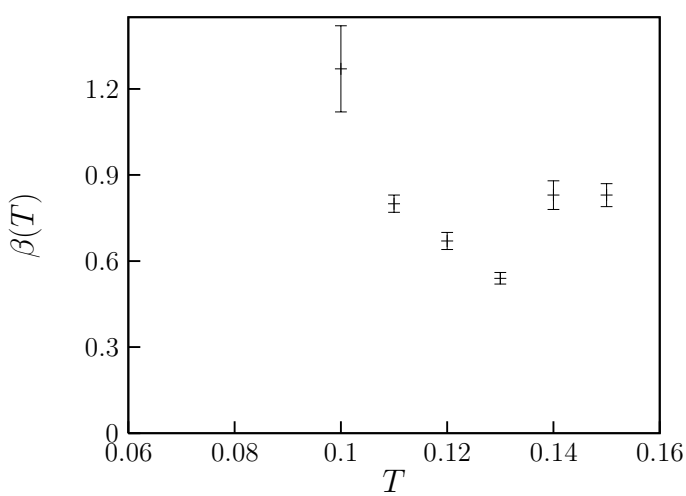

(b)

FIG. 4: Exponents (a) $\alpha$ and (b) $\beta$ versus temperature $T$ in the fit of the non-equilibrium relaxation for $t_{w}=0$ to the Ogielski form. 


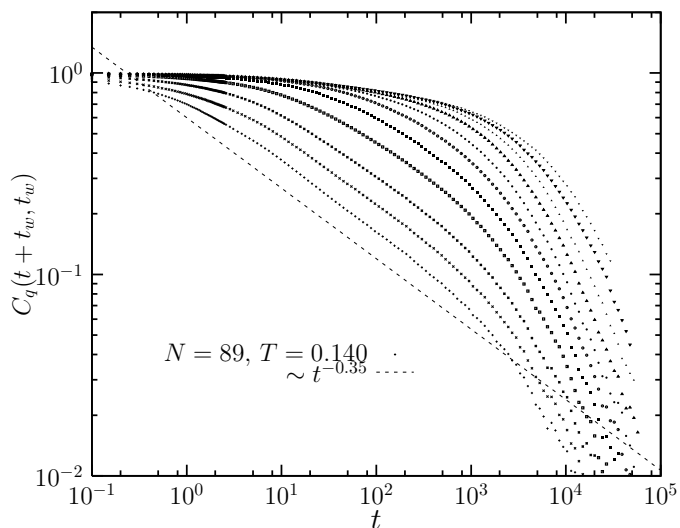

(a)

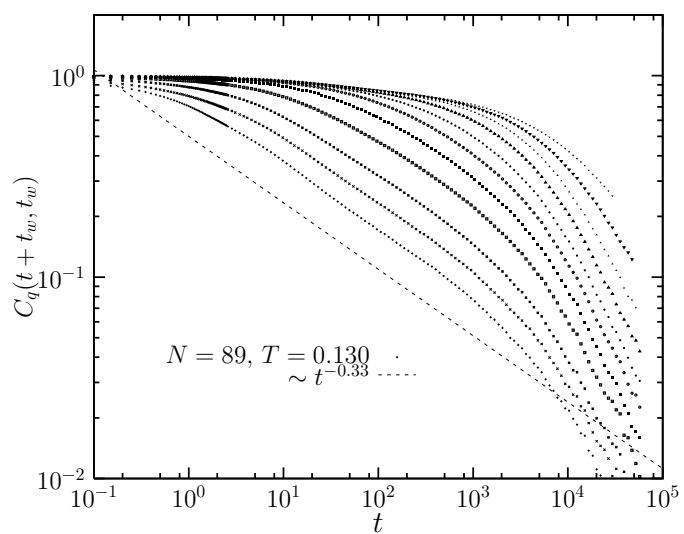

(b)

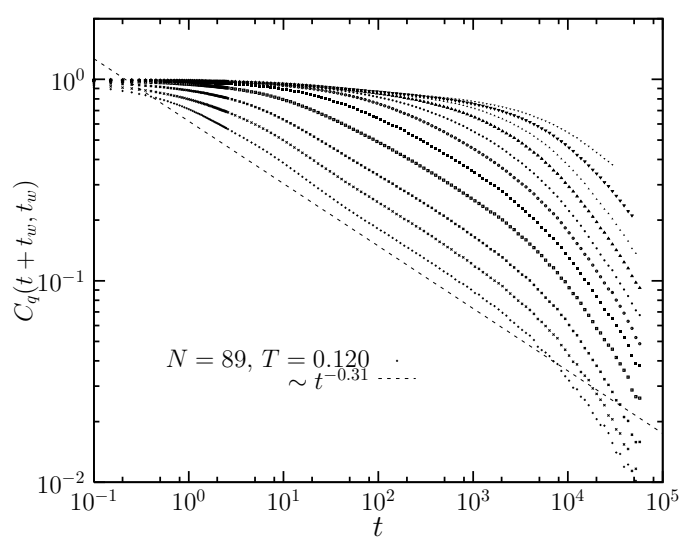

(c)

FIG. 5: Aging relaxation of the chirality autocorrelation function $C_{q}\left(t+t_{w}, t_{w}\right)$ for various values of the waiting time $t_{w}=$ $0,1,3,10,30,100,300,1000,3000,10000$, and 30000 (from left to right), starting from random initial states, at temperature $T=$ (a) 0.14, (b) 0.13, and (c) 0.12. Power-law behavior $t^{-\alpha}$ in the intermediate-time regime is manifested by dotted lines with the exponent $\alpha=$ (a) 0.35 , (b) 0.33 , and (c) 0.31 . As the waiting time grows, the relaxation develops simple stretched exponential behavior. 


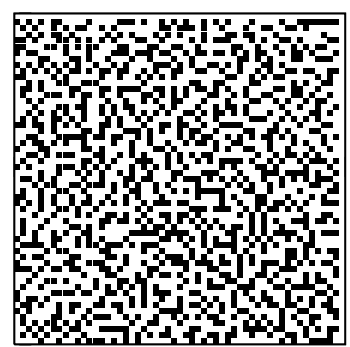

(a)

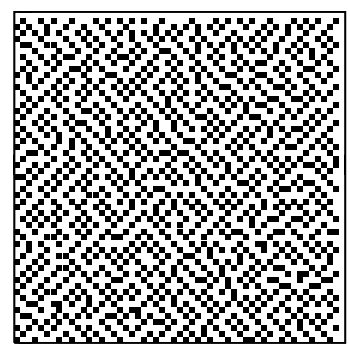

(c)

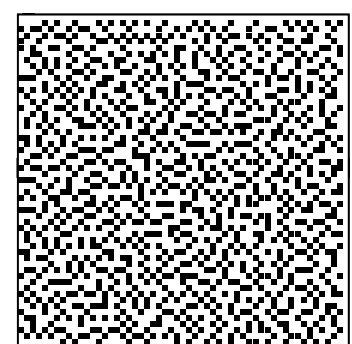

(b)

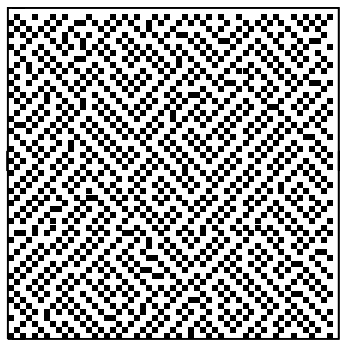

(d)

FIG. 6: Snapshots of the vortex configuration in the system of size $L=55$, beginning from a random initial state and quenched to temperature $T=0.130$, at time $t=$ (a) 0.0 , (b) 8.0, (c) 128, and (d) 2048. 


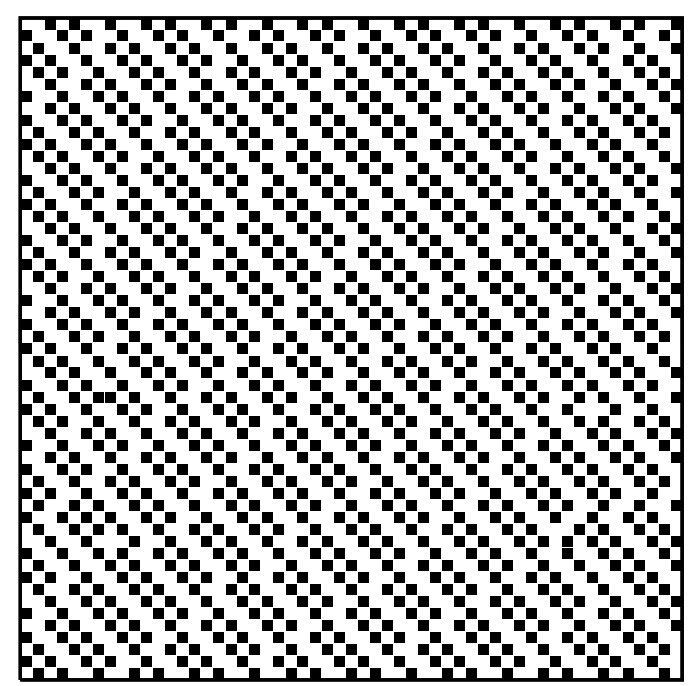

(a)

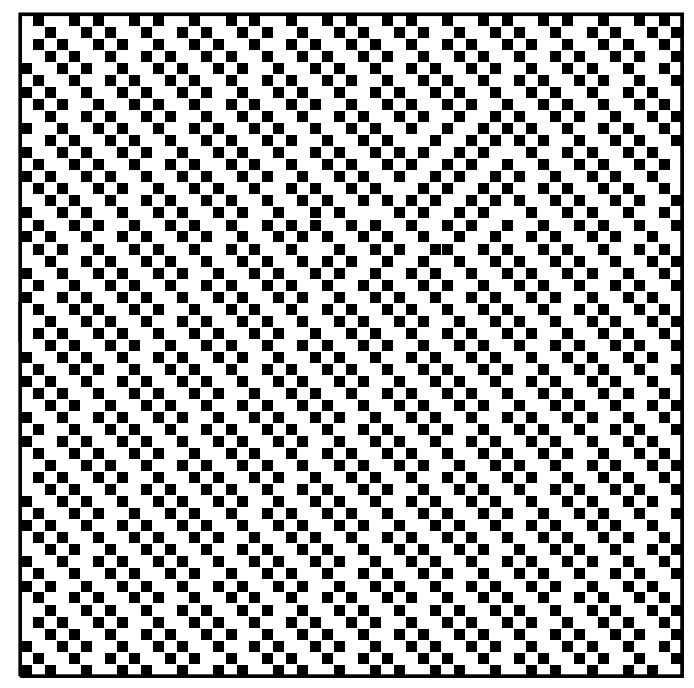

(b)

FIG. 7: Snapshots of the vortex configuration obtained via CSA for lattice size $L=55$ with energy $E=($ a) -1.273786 and (b) -1.274077 (in units of $\hbar I_{c} / 2 e$ ), respectively. 